\title{
Human face recognition methods based on principle component analysis (PCA), wavelet and support vector machine (SVM): a comparative study
}

Eman A. Gheni, Zahraa M. Algelal

Department of Computer Science, Faculty of Education for Girls, University of Kufa, Iraq

\begin{tabular}{l}
\hline Article Info \\
\hline Article history: \\
Received Feb 10, 2020 \\
Revised Apr 12, 2020 \\
Accepted Apr 24, 2020 \\
\hline Keywords: \\
Face recognition (FR) \\
ORL database \\
Principal component analysis \\
(PCA) \\
Support vector machines (SVM) \\
Wavelet transforms \\
Wavelet-PCA \\
\hline
\end{tabular}

\begin{abstract}
Human face Recognition systems are increasingly gaining more importance and can be utilized throughout many applications like video surveillance, Security, human-computer intelligent interaction, etc. this paper presents performance comparison between three feature extraction techniques for an automatic face recognition system. In the first step, we benefit from wavelet Transforms, Principal Component Analysis (PCA) and combining Wavelet with PCA as feature extracting methods. After feature vectors generation, linear and nonlinear Support Vector Machines (SVM) are usually used for implementing the classification or recognition step. These methods are compared on accuracy in an ORL database for face recognition applications including 400 images of 40 people.
\end{abstract}

Copyright $(0) 2020$ Institute of Advanced Engineering and Science. All rights reserved.

\section{Corresponding Author:}

Zahraa M. Algelal,

Department of Computer Science,

Faculty of Education for Girls,

University of Kufa, Najef, Iraq.

Email: zahraam.algelal@uokufa.edu.iq

\section{INTRODUCTION}

The face is considered as one of the most effective and important parts by which persons recognize each other. Compared with other characteristics such as iris/retinas, fingerprints and voices, taking a person's face images is very easy and can be acquired from a distance by a camera [1]. Therefore, Face recognition (FR) has become one of the most popular research domains in such varied fields as biometrics, pattern recognition and computer vision over the last several years because of its noninvasive nature, requires virtually small effort from the user and because it is people's primary technique of human identification despite the fact that other methods of recognition can be more accurate $[2,3]$.

FR is a very important technique in daily life, especially for security and surveillance fields, such as:-Law Enforcement, Access Control System, Passports, etc [4]. This technique may appear a simple function the for humans in changing of backgrounds, illuminations and appearances, and yet these systems still cannot achieve a completely reliable performance. The challenges arise because of the variation in face expressions, scale, pose, etc. [5].

Human face recognition system includes two main stages known as training and testing as shown in Figure 1, both involving comparable processing of initial stage. Preprocessing is considered as the most important step in any recognition system by enhancing input face image features in order to extract it. After preprocessing, the preprocessed image is presented to the feature extraction step and then the relevant features in the extracted feature vector are selected in the feature selection step. The training phase stores selected features in a gallery to be used later on, while testing phase compares the selected features from the test face image with the features from the gallery to recognize the face by using a suitable classifier $[5,6]$. 
There are two approaches of face recognition methods; Global matching approach and Local matching approach. Global matching methods characterized by using a single feature vector to perform the whole face and consider it as input to the recognition system. While in Local matching methods, several facial characteristics are determined to base the recognition on, then, comparing and integrating the corresponding local statistics to classify the faces in recognition results [7, 8]. Fortunately, face recognition methods have also developed, which employ different approaches such as PCA [9-11], wavelet transform based methods [12] and machine learning such as Convolution Neural Network (CNN) [13], Genetic Algorithms [14] and clustering [15, 16].

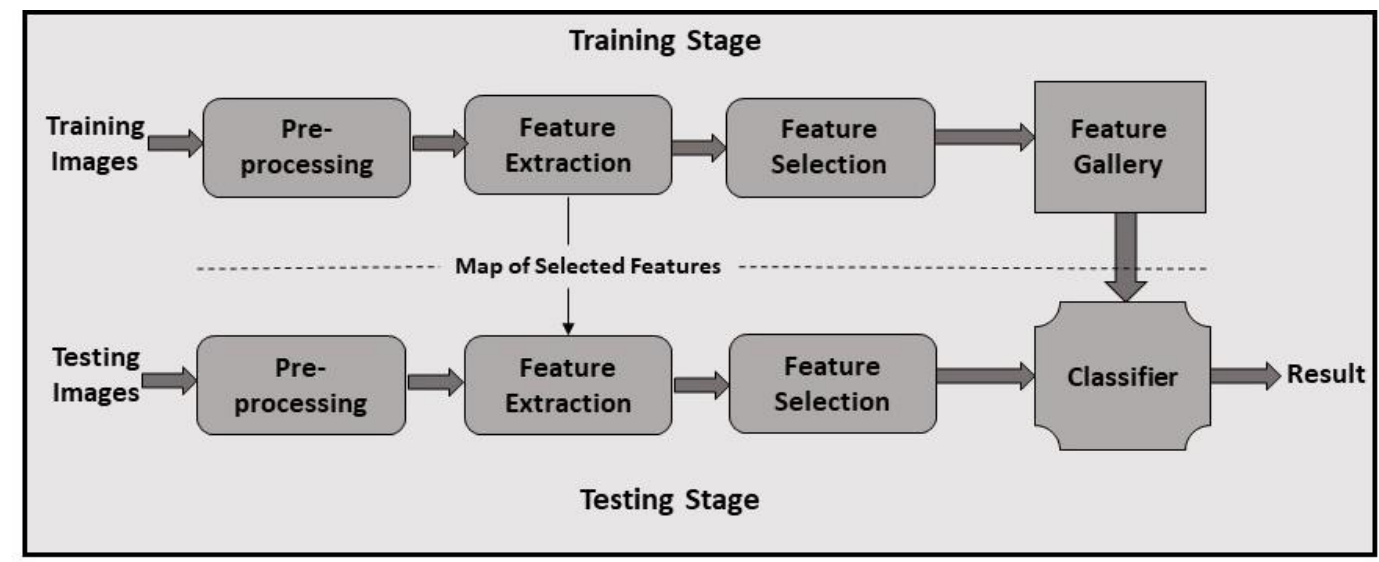

Figure 1. General diagram of a human face recognition system

The work shown in this paper adapts three methods (PCA, Wavelets and Wavelet-PCA) to extract features on the ORL face database as a first step. Then, output features will be used by both SVM (with linear and nonlinear kernels) for classification. The content of this study is organized into five sections as follows: materials most related to our work are represented in Section 2, Section 3 describing proposed research methods, Section 4 covers experimental results and proposed work analysis, and, Section 5 exhibits some conclusions from obtained results.

\section{RELATED WORK}

Many researchers have considered FR as headlines in their work; Most of differences between these works are restricted to feature extraction methods, its type and how it really functions. Some of their published works are as follows:

Rahul, et. al., [9] recognized faces using PCA+SVD. By using PCA method for face Reorganization along with the help of Eigen Vector (commonly called "Eigen Faces"), the test image is projected onto subspace. Euclidean distance will be measured by adopting this method for face recognition, all test images will be checked in this method. The Accuracy of face detection when taking various and numerous faces from training database will increase up to $30 \%$ when utilizing PCA+SVD if compare to using PCA alone.

Mahmood [10] presented his work in two main sections. Face recognition technique, consisting of four stages, including acquisition, preprocessing, features extraction (using PCA to extract best possible features that represents users' face image), and recognition, which uses Artificial feeding forward neural network with back propagation algorithm. While the second represents computers network system that consists of number of clients connected to web server on the internet. Face recognition is examined for three different cases, and delivered a recognition rate of $97.222 \%, 94.444 \%, 91.667 \%$ respectively, when applied on six persons in the first case, 12 persons in the second and 18 in the third while being connected to the server.

Jain and Bhati [12] presented a face recognition system that used a combination of wavelet transform, Principle component analysis and neural network. To obtain an image's analysis into different frequency components at different resolution scales, the wavelet transform can be used. These represent decomposition the image into four sub bands (LL, LH, HL, HH). LL represents the approximation contents and the other sub-bands represent details (edges), PCA is applied to the LL for extracting features. These features are used for training the classifier depending on artificial neural networks. Thus, recognition rate for various training and testing data sets will locate and identify the classifier's performance. 
Bahurupi and Chaudhari [11] presented a face recognition system consists of Principal Component Analysis (PCA) algorithm and Euclidean distance. In this case PCA is not only used to extract facial features, as an efficient method of feature extraction depends on face as global feature, but also works on successful reduction of source image dimensions, while keeping the essential data (features) contained in images. Finally, using distance measurement methods, like Euclidean distance is utilized to classify images' feature presented in test and database image.

\section{FEATURES EXTRACTION METHODS}

\subsection{Principal component analysis (PCA)}

PCA is also known as Eigenfaces method, which specializes in face images that was presented by Turk and Pentland [17] for face recognition. Through Eigenfaces method, every image in training set will decompose into smaller sets of feature vectors called "eigenfaces", provided from projecting image to the base in image space, aided by principal component analysis.

This approach can be described as follows:

a) Eigenvectors or eigenfaces of the covariance matrix are calculated. This is done by keeping only the $\mathrm{N}$ images correspond to highest eigenvalues from training face images. These $\mathrm{N}$ images describe the "face space".

b) These eigenvectors end up being eigenvalue by calculating the corresponding distribution in $\mathrm{N}$-dimensional weight space for each known person by projecting face image onto the "face space"

Recognition phase is implemented by comparing the position of a new face image with identified persons in the "face space".

\subsection{Wavelet transform}

Wavelet transform method is currently utilized in many studies in biometric recognition such as palm, iris, fingerprint and face where it has been used more widely than the other popular methods for face recognition [18]. Wavelet transform in FR is intuitive that images of a given person captured under different lighting conditions may differ significantly, which influences the accuracy of face recognition [10]. Discrete Wavelet Transform (DWT) is a type of wavelet transform suitable for computer implementation and is usually classified into 1-dimensional and 2-dimensional transform. Some of the basic types of wavelets used in DWT are Haar, Morlet, Mallat, Meyer, etc [19].

In an image (two-dimensional signal), low frequency content provides overall description while the high frequency content provides more detailed information. By implementing wavelet transform, original image is decomposed into four different sub-bands, specifically, LL (low-low), LH (low-high), HL (high-low) and HH (high-high) bands. The band LL is a coarser approximation of the image. The band LH gives vertical features where its coefficients are highly sensitive to variations in expression, and the band HL gives horizontal features where its coefficients differs with pose changes in the pose. The band HH gives diagonal features where its coefficients are sensitive to noise [20, 21]. After performing 1-level DWT, an image $(\mathrm{G})$ can be represented with its sub-bands according to the (1).

$$
G=G_{a}^{1}+\left\{G_{h}^{1}+G_{v}^{1}+G_{d}^{1}\right\}
$$

For data dimensional reduction, DWT can be used an $\mathrm{N}$ number of times to have an $\mathrm{N}$-level decomposition, and an image is represented according to the expression (2), Figure 2 shows an example of the three- level 2D-DWT decomposition process [22].

$$
I=G_{a}^{N}+\sum_{i=1}^{N}\left\{I G_{h}^{1}+G_{v}^{1}+G_{d}^{1}\right\}
$$

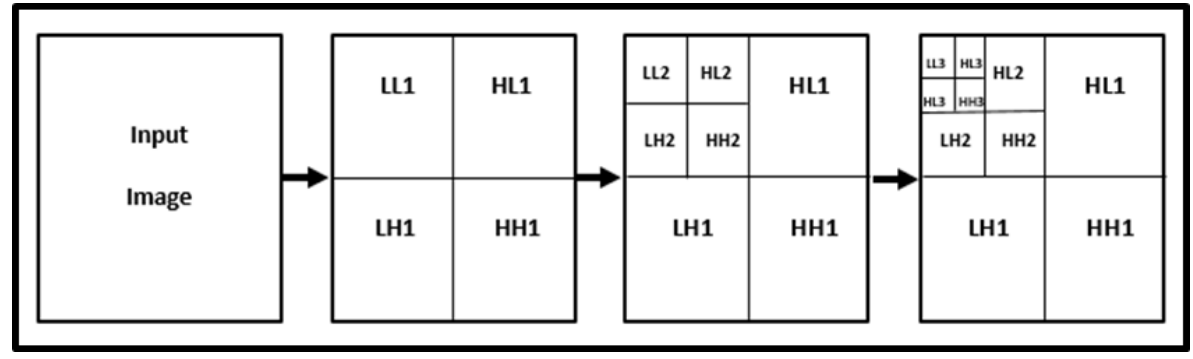

Figure 2. A 3- level 2D-wavelet decomposition on the input image 


\section{CLASSIFICATION METHOD}

\subsection{Support vector machine (SVM)}

SVMs is a statistical model suggested by Vapnik [23], which tries to search an Optimal Separating Hyperplane (OSH) that enables linear separation of data sets from various classes (Figure 3 ). It is also able to capture non-linearities in data by using kernel functions that transforms data into new space and then finds the OSH.

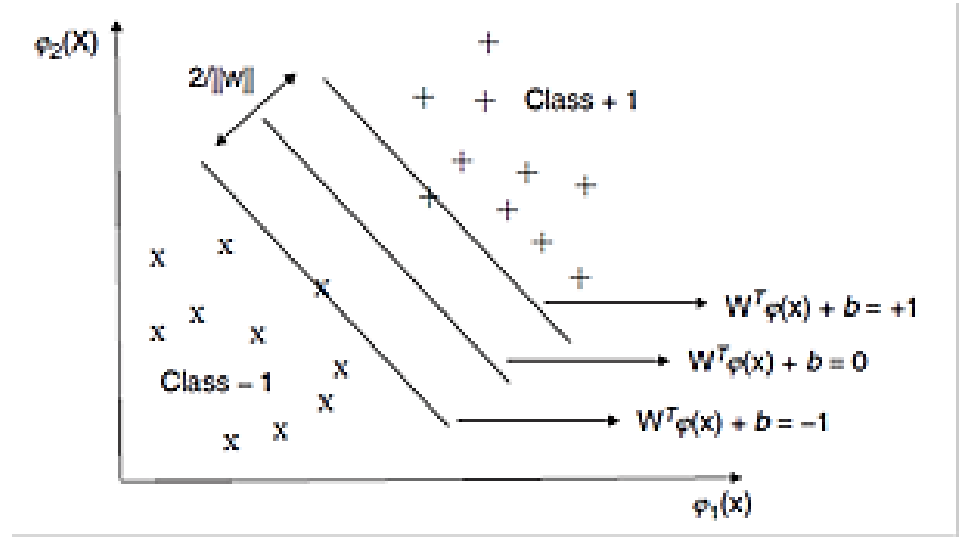

Figure 3. Illustration of SVM optimization of separate the data sets

Given a training set $x_{i} \in I R^{n} i=1,2, \ldots, N$ and corresponding binary class $y_{i} \in\{-1,1\}$, in the linearly separable case it can recognize two classes and the OSH separating function turns into the form:

$$
\begin{aligned}
& w^{T} * \phi\left(x_{i}\right)+\mathrm{b} \geq 1, \quad y_{i}=1 \\
& w^{T} * \phi\left(x_{i}\right)+\mathrm{b} \leq-1, \quad y_{i}=-1
\end{aligned}
$$

which is equivalent to

$$
y_{i} *\left[w^{T} * \phi\left(x_{i}\right)+\mathrm{b}\right] \geq 1, \quad \mathrm{i}=1, \ldots, 1
$$

To apply nonlinear separation of the data sets, SVM utilizes the non-linear function $\phi(\cdot)$ to transform data into a higher dimensional feature space where a hyperplane can be used for separation. In a high dimensional space realization of $\left.\left\langle\phi\left(x_{i}\right)\right\rangle * \phi(x)\right)$ multiplication is intractable. For that, "Kernel Functions" in $\left.k\left(x_{i}, x\right)=\left\langle\phi\left(x_{i}\right)\right\rangle * \phi(x)\right)$ form are used. In such processes there are some commonly used kernel functions:

(i) linear kernel

$$
k\left(x_{i}, x\right)=x_{i} * x
$$

(ii) Polynomial Kernel Function

$$
k\left(x_{i}, x\right)=\left(x_{i} * x+1\right)^{p}
$$

(iii) RBF Kernel Function

$$
k\left(x, x_{i}\right)=\exp \left[-\gamma\left\|x-x_{i}\right\|^{2}\right]
$$

Linear and polynomial Kernel Function classification performance was examined in this study.

\section{EXPERIMENTAL RESULTS}

The performance of each feature extraction method was evaluated using the ORL face database [24] with both linear and non-linear SVM under pose and sample size variating conditions. Features were 
extracted using PCA, HAAR Wavelet and Wavelet-PCA techniques with Matlab (2013) coded program, "OSU Support Vector Machines Toolbox v3.00" [25] was used in Matlab to implement SVM. (A personal computer supporting Intel(R) core2 Duo 2.2 GHZ CPU and 2 GB RAM was used during the experiment.

400 images of 40 individual's faces (each individual had 10 different poses) were contained in ORL database, different conditions were taken into consideration (expression, illumination, etc.). Each image was of $112 \times 92$ pixels in size, with 256 grey levels. Seven training sets were composed, each included the first nine image samples for each person in this experiment. For each person, our training sets included images 3 , 4, 5, 6, 7, 8 and 9, and remaining images were used for testing. i.e. The first three poses of each person are used for training in the first case, and the remaining seven poses were used for testing. In the second case, the first four poses of each person are used for training and the remaining six poses are used for testing and so on for remaining test cases.

Experiment was performed by utilizing different numbers of eigenfaces (first 20, 30, 40, $\ldots, 120$ elements of each feature vector) were used in PCA method for features extraction, These PCA features vectors were used for classification. For Wavelet Transform (WT) method, we extract feature vectors using a HAAR wavelet function. Experiment was performed on first four levels of wavelet transformation. Starting with $112 \times 92$ pixels face image size, then decomposed to $(56 \times 64,28 \times 23,14 \times 12,7 \times 6)$ pixels on the wavelet level 1, 2, 3 and 4, respectively. Wavelet-PCA for feature extraction was used in the third experiment. Up to three decomposition levels for images (that supports highest accuracy) using HAAR wavelet transform. Low frequency band (LL) at 3th level was processed using PCA with same eigenfaces numbers (first 20,30,40, ., 120 elements of each feature vector). This procedure of three feature extraction methods with both linear and non-linear SVM was used in the experiment, choosing "Cost of the constrain violation" parameter as 1.0. and an exponent value was set to 1 for linear and 3 for polynomial forms during SVM classification.

\subsection{Experiment 1}

PCA was used for feature vectors extraction in the first experiment (with variable eigenfaces numbers), SVM was used afterwards to classify these feature vectors. (92.7\%) was the highest average of face recognition accuracy with 40, 50 and 60 features in linear SVM,. No significant improvement in the recognition rate was observed for higher number of eigenvectors. Table 1 exhibits these results, it is also noticeable that training images number per person would significantly increase recognition's accuracy. A peak of $(97.5 \%)$ in recognition rate is obtained in case of 9 images per person.

Table 2 illustrates the recognition rates of non-linear SVM classifier (polynomial kernel) by varying the eigenfaces and maximum average recognition rate obtained is $(91.5 \%)$ with 40 features, the accuracy degradation when using the feature vector length is larger than 40. Comparison of average recognition rates for both linear and non-linear SVM is displayed in Figure 4, the results show that linear SVM has higher recognition accuracy.

Table 1. Recognition rates using PCA with linear SVM

\begin{tabular}{ccccccccccccc}
\hline \multirow{2}{*}{$\begin{array}{c}\text { Training } \\
\text { samples/class }\end{array}$} & \multicolumn{8}{c}{ Face recognition accuracy (\%) } \\
\hline & 20 & 30 & 40 & 50 & 60 & 70 & 80 & 90 & 100 & 110 & 120 \\
\hline 3 & 80.4 & 83.9 & 85.0 & 84.6 & 84.6 & 83.6 & 84.3 & 84.6 & 84.3 & 84.6 & 84.6 \\
4 & 85.8 & 88.8 & 88.8 & 87.9 & 87.1 & 87.1 & 87.1 & 87.9 & 87.9 & 87.9 & 87.9 \\
5 & 87.0 & 88.5 & 89.5 & 90.0 & 89.5 & 89.5 & 88.5 & 89.0 & 88.5 & 89.0 & 89.0 \\
6 & 95.0 & 95.6 & 95.6 & 95.6 & 96.3 & 96.9 & 95.6 & 95.0 & 95.0 & 95.6 & 95.6 \\
7 & 95.0 & 95.8 & 95.0 & 95.8 & 96.7 & 96.7 & 96.7 & 97.5 & 96.7 & 96.7 & 96.7 \\
8 & 96.3 & 96.3 & 97.5 & 97.5 & 97.5 & 96.3 & 97.5 & 97.5 & 96.3 & 96.3 & 96.3 \\
9 & 95.0 & 95.0 & 97.5 & 97.5 & 97.5 & 97.5 & 97.5 & 97.5 & 97.5 & 97.5 & 97.5 \\
average & 90.6 & 92.0 & 92.7 & 92.7 & 92.7 & 92.5 & 92.5 & 92.7 & 92.3 & 92.5 & 92.5 \\
\hline
\end{tabular}

Table 2. Recognition rates using PCA and non-linear SVM classifier with a polynomial kernel

\begin{tabular}{cccccccccccc}
\hline \multirow{2}{*}{$\begin{array}{c}\text { Training } \\
\text { samples/class }\end{array}$} & \multicolumn{8}{c}{ Face recognition accuracy (\%) } \\
\hline 3 & 20 & 30 & 40 & 50 & 60 & 70 & 80 & 90 & 100 & 110 & 120 \\
4 & 79.3 & 82.1 & 83.6 & 54.6 & 52.5 & 51.8 & 50.4 & 50.4 & 49.6 & 49.3 & 49.6 \\
5 & 83.8 & 85.0 & 85.8 & 65.4 & 64.2 & 63.8 & 62.5 & 61.7 & 60.4 & 60.8 & 60.4 \\
6 & 88.5 & 89.5 & 88.5 & 71.5 & 70.0 & 67.5 & 67.0 & 66.5 & 66.5 & 66.5 & 66.5 \\
7 & 95.6 & 95.0 & 95.0 & 85.0 & 83.8 & 81.9 & 81.3 & 80.6 & 79.4 & 79.4 & 78.8 \\
8 & 95.0 & 96.7 & 96.7 & 90.0 & 88.3 & 87.5 & 87.5 & 87.5 & 86.7 & 86.7 & 85.8 \\
9 & 95.0 & 95.0 & 96.3 & 92.5 & 91.3 & 91.3 & 91.3 & 90.0 & 90.0 & 90.0 & 90.0 \\
average & 95.0 & 95.0 & 95.0 & 90.0 & 90.0 & 90.0 & 90.0 & 90.0 & 90.0 & 90.0 & 90.0 \\
\hline
\end{tabular}

Human face recognition methods based on principle component analysis (PCA), wavelet... (Eman A. Gheni) 


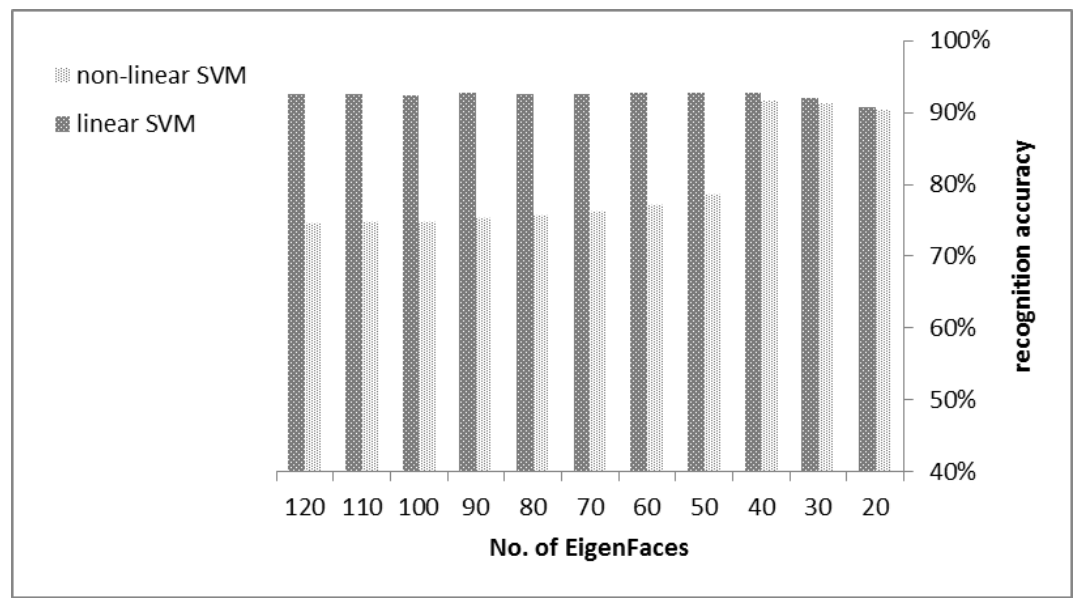

Figure 4. Comparison of linear and non-linear SVM classification accuracy with PCA

\subsection{Experiment 2}

Feature vectors were extracted using HAAR wavelet function in the second experiment. The image is decomposed at four levels. Four sub-bands were obtained at each level of wavelet transformation: LL, LH, HL, and HH, the sub-band LL was used as the input to the next level of decomposition. The sub band LL1, LL2 LL3, and LL4 were classified using SVM. (93.1\%) was the highest average of face recognition accuracy at 3rd level of decomposition in linear SVM. Experiment results are explained in Table 3.

Eecognition rates of non-linear SVM classifier (polynomial kernel) are illustrated in Table 4, and $(92.4 \%)$ is the maximum average recognition rate obtained at 3rd level of decomposition, the more image decomposition took place, accuracy degraded. Comparison of average recognition rates for both linear and non-linear SVM is shown in Figure 5, results explain that linear SVM has higher recognition accuracy.

Table 3. Recognition rates using wavelet and linear SVM

\begin{tabular}{cccccc}
\hline & \multicolumn{5}{c}{ Face recognition accuracy (\%) } \\
Training samples/class & L1 & L2 & L3 & L4 & L5 \\
\hline 3 & 85.0 & 86.1 & 87.5 & 81.8 & 74.3 \\
4 & 87.9 & 88.8 & 90.8 & 86.7 & 79.6 \\
5 & 88.5 & 89.0 & 91.5 & 91.0 & 86.5 \\
6 & 95.0 & 95.0 & 96.3 & 96.9 & 93.8 \\
7 & 95.8 & 95.8 & 95.8 & 97.5 & 94.2 \\
8 & 96.3 & 95.0 & 95.0 & 97.5 & 95.0 \\
9 & 97.5 & 97.5 & 95.0 & 97.5 & 95.0 \\
Average & 92.3 & 92.5 & 93.1 & 92.7 & 88.3 \\
\hline
\end{tabular}

Table 4. Recognition rates using wavelet and non-linear SVM classifier with a polynomial kernel

\begin{tabular}{cccccc}
\hline \multirow{2}{*}{ Training samples/class } & \multicolumn{5}{c}{ Face recognition accuracy (\%) } \\
& L1 & L2 & L3 & L4 & L5 \\
\hline 3 & 84.3 & 84.6 & 83.6 & 77.5 & 72.5 \\
4 & 87.9 & 89.6 & 87.9 & 83.8 & 77.5 \\
5 & 88.5 & 90.0 & 91.5 & 92.0 & 86.0 \\
6 & 95.6 & 96.3 & 96.9 & 96.3 & 93.1 \\
7 & 95.8 & 95.8 & 95.8 & 96.7 & 95.0 \\
8 & 96.3 & 95.0 & 96.3 & 96.3 & 95.0 \\
9 & 95.0 & 95.0 & 95.0 & 95.0 & 92.5 \\
Average & 91.9 & 92.3 & 92.4 & 91.1 & 87.4 \\
\hline
\end{tabular}




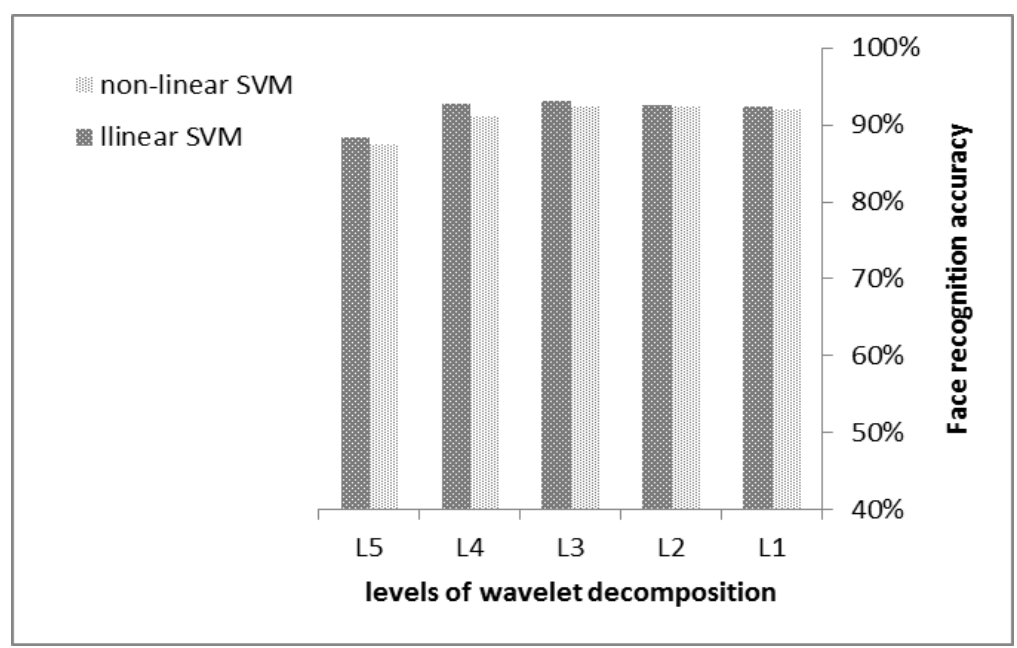

Figure 5. Comparison of linear and non-linear SVM classification accuracy with wavelet transformation

\subsection{Experiment 3}

Feature vectors are extracted using Wavelet-PCA in the third experiment, decomposition of up to three levels was used on source images (that have highest accuracy), while PCA was used to process Low frequency band (LL) at 3rd level, SVM was utilized to classify resulting feature vectors. (93.4\%) was the highest average of face recognition accuracy in linear SVM at 3rd level of decomposition, and the feature vector length is 60 . Table 5 exhibits the results. Recognition rates of non-linear SVM classifier (polynomial kernel) is illustrated in Table $6,(84.6 \%)$ was the maximum average recognition rate obtained at the feature vector length is 40 , when feature vector length larger than 40 or 60 was used, accuracy degraded. Comparison of average recognition rates for both linear and non-linear SVM are shown in Figure 6, results show that linear SVM has higher classification accuracy. General results demonstrating the efficiency of using of Wavelet Transformation method over PCA and Wavelet-PCA methods in face recognition are shown in Figure 7.

Table 5. Recognition rates (\%) using wavelet-PCA and linear SVM classifier

\begin{tabular}{|c|c|c|c|c|c|c|c|c|c|c|c|}
\hline \multirow{2}{*}{ Training samples/class } & \multicolumn{11}{|c|}{ No. of Eigen Faces } \\
\hline & 20 & 30 & 40 & 50 & 60 & 70 & 80 & 90 & 100 & 110 & 120 \\
\hline 3 & 82.5 & 86.8 & 86.4 & 87.1 & 87.9 & 87.9 & 87.5 & 87.5 & 87.5 & 87.5 & 87.5 \\
\hline 4 & 85.4 & 88.3 & 90.8 & 90.4 & 90.8 & 90.8 & 90.8 & 90.8 & 90.8 & 90.8 & 90.8 \\
\hline 5 & 90.0 & 91.0 & 91.5 & 91.5 & 92.0 & 91.5 & 91.5 & 92.0 & 92.0 & 92.0 & 91.5 \\
\hline 6 & 95.0 & 95.6 & 96.9 & 96.3 & 96.3 & 96.3 & 96.3 & 96.3 & 96.3 & 96.3 & 96.3 \\
\hline 7 & 94.2 & 95.0 & 95.8 & 95.8 & 95.8 & 95.8 & 95.8 & 95.8 & 95.8 & 95.8 & 95.8 \\
\hline 8 & 96.3 & 95.0 & 95.0 & 95.0 & 96.3 & 95.0 & 95.0 & 95.0 & 95.0 & 95.0 & 95.0 \\
\hline 9 & 95.0 & 92.5 & 95.0 & 95.0 & 95.0 & 95.0 & 95.0 & 95.0 & 95.0 & 95.0 & 95.0 \\
\hline Average & 91.2 & 92.0 & 93.1 & 93.0 & 93.4 & 93.2 & 93.1 & 93.2 & 93.2 & 93.2 & 93.1 \\
\hline
\end{tabular}

Table 6. Recognition rates (\%) using wavelet-PCA and non-linear SVM classifier with a polynomial kernel

\begin{tabular}{|c|c|c|c|c|c|c|c|c|c|c|c|}
\hline \multirow{2}{*}{ Training samples/class } & \multicolumn{11}{|c|}{ No. of Eigen Faces } \\
\hline & 20 & 30 & 40 & 50 & 60 & 70 & 80 & 90 & 100 & 110 & 120 \\
\hline 3 & 68.6 & 68.2 & 68.9 & 69.3 & 68.9 & 68.6 & 68.6 & 67.9 & 67.9 & 67.9 & 67.9 \\
\hline 4 & 75.8 & 74.2 & 73.3 & 74.2 & 73.8 & 73.8 & 73.8 & 73.8 & 73.8 & 73.8 & 73.8 \\
\hline 5 & 82.0 & 79.0 & 79.0 & 78.5 & 79.5 & 79.0 & 79.0 & 79.0 & 79.0 & 79.0 & 78.5 \\
\hline 6 & 92.5 & 91.3 & 91.3 & 91.3 & 91.3 & 91.3 & 91.3 & 91.3 & 91.3 & 91.3 & 91.3 \\
\hline 7 & 95.0 & 94.2 & 93.3 & 93.3 & 93.3 & 93.3 & 93.3 & 93.3 & 93.3 & 93.3 & 93.3 \\
\hline 8 & 95.0 & 93.8 & 93.8 & 92.5 & 92.5 & 93.8 & 92.5 & 92.5 & 92.5 & 92.5 & 92.5 \\
\hline 9 & 92.5 & 90.0 & 92.5 & 92.5 & 92.5 & 92.5 & 92.5 & 92.5 & 92.5 & 92.5 & 92.5 \\
\hline Average & 85.9 & 84.4 & 84.6 & 84.5 & 84.5 & 84.6 & 84.4 & 84.3 & 84.3 & 84.3 & 84.2 \\
\hline
\end{tabular}




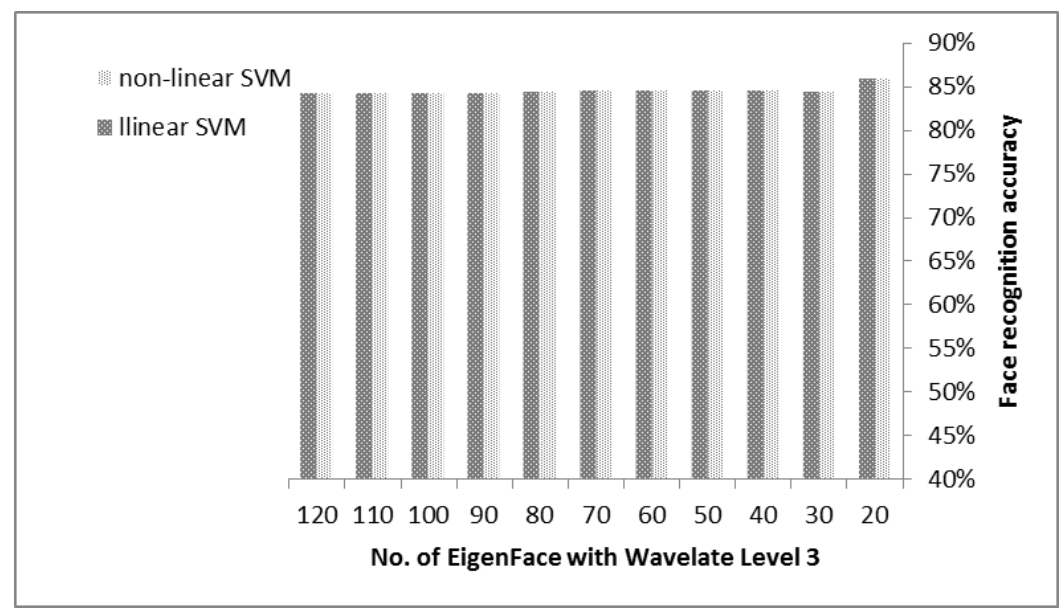

Figure 6. Comparison of linear and non-linear SVM classification accuracy with Wavelet-PCA

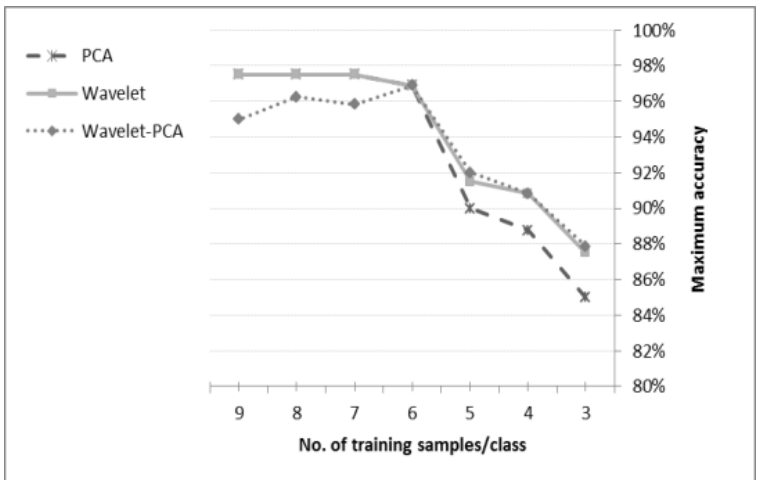

(a)

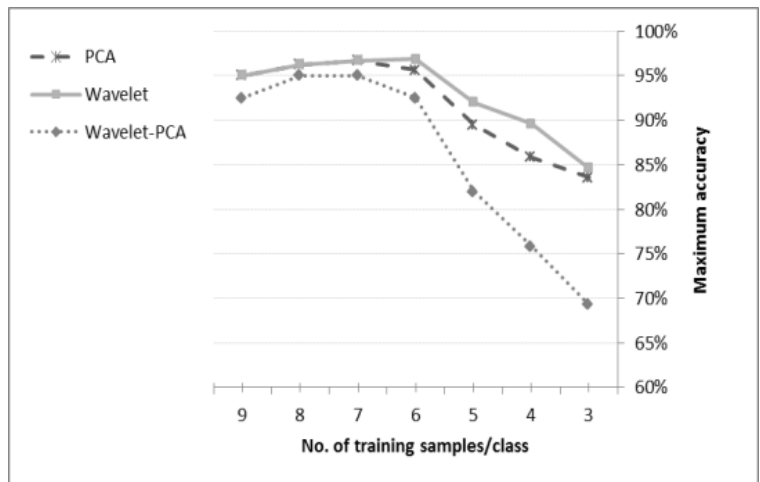

(b)

Figure 7. Comparison of classification accuracy variance with the number of training samples per class for (a) Linear SVM, (b) Non-linear SVM

\section{CONCLUSION}

The main focus of this study was on performance comparison of three feature extraction approaches (PCA, Wavelets and Wavelet-PCA) using 400 face images of 40 people from ORL face database. SVM (with linear and nonlinear of kernels) were applied by using the extracted features vectors for recognition phase. Different number of training images to compare recognition rates of these methods was used to implement this experiment, 93.4\% was the best average recognition rate achieved with Wavelet-PCA (in linear SVM). It was found that accuracy enhancements were very limited by compressing with PCA or WT alone, while WT method over PCA and Wavelet-PCA methods in non- linear SVM was demonstrated by simulation results.

\section{REFERENCES}

[1] A. A. M. Al-Aesawy, "Human Face Detection and Tracking By using Skin Color Molding and Component Operators Connecting," M.Sc. thesis, AL-Mustanseryah University, Bagdad, Iraq, 2006.

[2] X. Zhang and Y. Gao, "Face recognition across pose: a review," Pattern Recognition, vol. 42, no. 11, pp. 2876-2896, 2009.

[3] N. Kar, et al., "Study of implementing automated attendance system using face recognition technique," International Journal of computer and communication engineering, vol. 1, no. 2, pp. 100-103, 2012.

[4] S. Sakthivel and R. Lakshmipathi, "Enhancing Face Recognition Using Improved Dimensionality Reduction and Feature Extraction Algorithms-An Evaluation With ORL Database," International Journal of Engineering Science and Technology, vol. 2, no. 6, pp. 2288-2295, 2010.

[5] E. A. Gheni, et al., "Model-Based Active Appearance Model Approach for Face Recognition," Journal of Engineering and Applied Sciences, vol. 14, no. 9, pp. 2988-2992, 2019. 
[6] N. M. Rao, et al., "Face recognition using entropy based feature enhancement and Parallel Dual Pose testing," in 2014 International Conference on Medical Imaging, m-Health and Emerging Communication Systems (MedCom), pp. 352-357, 2014.

[7] T. F. Karim, et al., "Face recognition using PCA-based method," in 2010 IEEE International Conference on Advanced Management Science (ICAMS 2010), pp. 158-162, 2010.

[8] T. Ahonen, et al., "Face recognition with local binary patterns," in European conference on computer vision, pp. 469-481, 2004.

[9] R. Jain and S. Kumar, "High accuracy face reorganization by pca - svd," International Journal of Engineering Sciences \& Research Technology, vol. 5, no. 11, pp. 5-10, 2016.

[10] T. M. Mahmood, "Face recognition technique for network authentication," M.Sc. thesis, Al-Nahrain University, Bagdad, Iraq, 2014.

[11] S. P. Bahurupi and D. S. Chaudhari, "Principal component analysis for face recognition," International Journal of Engineering and Advanced Technology, vol. 1, no. 5, pp. 91-94, 2012.

[12] S. Jain and D. Bhati, "Face Recognition Using ANN with Reduce Feature by PCA in Wavelet Domain," International Journal of Scientific Engineering and Technology, vol. 2, no. 6, pp. 595-599, 2013.

[13] N. A. B. M. Kasim, et al., "Celebrity Face Recognition using Deep Learning," Indonesian Journal of Electrical Engineering and Computer Science, vol. 12, no. 2, pp. 476-481, 2018.

[14] N. A. A. Rahim, et al., "The Application of Modified Least Trimmed Squares with Genetic Algorithms Method in Face Recognition," Indonesian Journal of Electrical Engineering and Computer Science, vol. 8, no. 1, pp. 154-158, 2017.

[15] J. Lu, et al., "A method of face recognition based on fuzzy c-means clustering and associated sub-NNs," IEEE Transactions on Neural Networks, vol. 18, no. 1, pp. 150-160, 2007.

[16] A. Bhat, "K-medoids clustering using partitioning around medoids for performing face recognition," International Journal of Soft Computing, Mathematics and Control, vol. 3, no. 3, pp. 1-12, 2014.

[17] M. A. Turk and A. P. Pentland, "Face recognition using eigenfaces," in Proceedings of 1991 IEEE Computer Society Conference on Computer Vision and Pattern Recognition, pp. 586-591, 1991.

[18] J. Zhang, et al., "Comparison of wavelet, Gabor and curvelet transform for face recognition," Optica Applicata, vol. 41, no. 1, pp. 183-193, 2011.

[19] Z. S. Ahmed, "Palmprint Recognition with Statistical, Wavelet and Local Feature Extraction Methods," Doctoral dissertation, Eastern Mediterranean University (EMU)-Doğu Akdeniz Üniversitesi (DAÜ), 2015.

[20] V. Vidya, et al., "Face recognition using threshold based DWT feature extraction and selective illumination enhancement technique," Procedia Technology, vol. 6, pp. 334-343, 2012.

[21] A. N. Khedair, "Human face recognition using principal component analysis with wavelet and radon transforms," M.Sc. thesis, Al-Nahrain University, Bagdad, Iraq, 2006.

[22] E. Gumus, et al., "Evaluation of face recognition techniques using PCA, wavelets and SVM," Expert Systems with Applications, vol. 37, no. 9, pp. 6404-6408, 2010.

[23] V. Vapnik, "Statistical Learning Theory,” New York, John Willey \& Sons, 1998.

[24] ORL Face Database, "The database of faces, Digital Technology Group," Cambridge University Computer Laboratory. Available: http://www.cl.cam.ac.uk/research/dtg/attarchive/facedatabase.html.

[25] J. Ma and Y. Zhao, "OSU Support Vector Machines Toolbox, version 3.0," 2004. Available: http://www.csie.ntu.edu.tw/cjlin/libsvm. 